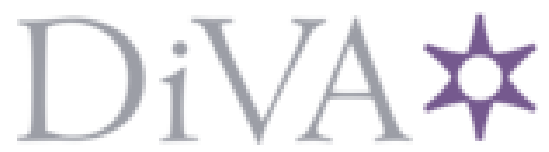

http://www.diva-portal.org

\title{
Preprint
}

This is the submitted version of a paper presented at 2017 IEEE/RSJ International Conference on Intelligent Robots and Systems (IROS).

Citation for the original published paper:

Almeida, D., Karayiannidis, Y. (2017)

Dexterous manipulation by means of compliant grasps and external contacts.

In: (pp. 1913-1920). IEEE

N.B. When citing this work, cite the original published paper.

Permanent link to this version:

http://urn.kb.se/resolve?urn=urn:nbn:se:kth:diva-215234 


\section{Dexterous Manipulation with Compliant Grasps and External Contacts}

\author{
Diogo Almeida
}

\begin{abstract}
We propose a method that allows for dexterous manipulation of an object by exploiting contact with an external surface. The technique requires a compliant grasp, enabling the motion of the object in the robot hand while allowing for significant contact forces to be present on the external surface. We show that under this type of grasp it is possible to estimate and control the pose of the object with respect to the surface, leveraging the trade-off between force control and manipulative dexterity. The method is independent of the object geometry, relying only on the assumptions of type of grasp and the existence of a contact with a known surface. Furthermore, by adapting the estimated grasp compliance, the method can handle unmodelled effects. The approach is demonstrated and evaluated with experiments on object pose regulation and pivoting against a rigid surface.
\end{abstract}

\section{INTRODUCTION}

Robots are often required to manipulate objects. They may have to pick-and-place a product from a conveyor belt to a storage box; they may be required to use a tool to execute some painting or welding task, or execute repetitive assembly steps in an assembly line. In typical industrial contexts, the robot will perform its task by employing parallel grippers to grasp an object. Once the object is grasped by the robot, it will stay rigidly attached to the gripper until the task is executed successfully. Such a grasp allows for an easier task accomplishment, as the robot does not need to account for an independent object movement, but prevents a dexterous tool usage.

Unlike robots, humans employ a wide array of dexterous motions with the grasped object. These dexterous motions allows us to attain a certain goal without excessive and awkward arm motion, and become more prominent in tasks where the object itself has to follow a complex trajectory, e.g., writing with a pen. The ability to relocate an object with respect to the grasping end-effector can be denominated as dexterous manipulation, or manipulative dexterity [1], [2]. Researchers have studied dexterous manipulation for years, where a three-fingered hand is considered to have the minimum amount of degrees of freedom that allow for a robot to manipulate an object in its grasp, i.e., to perform in-hand manipulation.

The authors are with the Computer Vision and Active Perception Lab. Centre for Autonomous Systems, School of Computer Science and Communication, Royal Institute of Technology KTH, SE-100 44 Stockholm, Sweden. e-mail: \{diogoa|yiankar\}akth.se

Y. Karayiannidis is with the Dept. of Signals and Systems, Chalmers University of Technology, SE-412 96 Gothenburg, Sweden, e-mail: yiannis@chalmers.se

This work has been carried out in the SARAFun project, partially funded by the EU within H2020 (H2020-ICT-2014/H2020-ICT-2014-1) under grant agreement no. 644938

\author{
Yiannis Karayiannidis
}

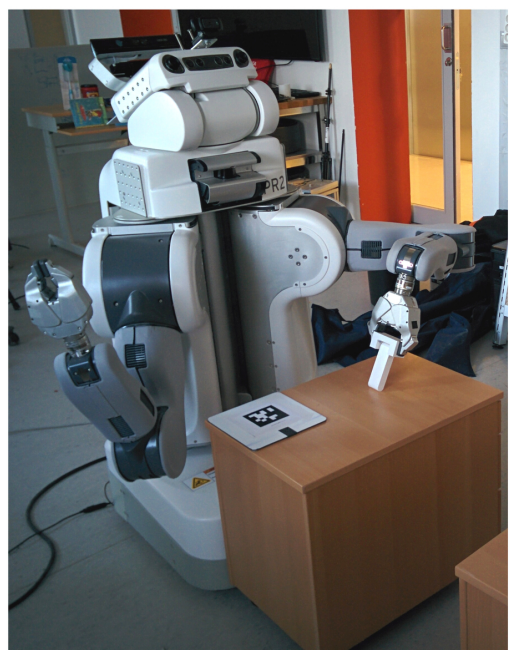

Fig. 1: PR2 robot manipulating an object under a compliant grasp.

Given that the majority of robotic manipulators employ parallel grippers, unable by design to perform in-hand manipulation, an alternative way to achieve dexterous manipulation is via extrinsic dexterity [3], i.e., by exploiting elements external to the robot. Pushing a grasped object against an external surface is a viable way of reorienting it; loosening the grasp on an object allows for its pose to change due to the force of gravity and, by using the arm dynamics, arbitrary forces can be exerted on the object, allowing its motion with respect to the gripper. It can be readily observed that humans employ both in-hand manipulation ('intrinsic dexterity') as well as extrinsic dexterity in order to manipulate objects. A simple manipulation primitive that can be easily accomplished through extrinsic dexterity is the pivoting of an object grasped by a parallel gripper. If the proper force is applied on the object, it will rotate (pivot) around the pinching point. Humans will often employ pivoting when reorienting objects in their grasp, often combined with other types of dexterous manipulation primitives.

In our present work we explore the idea of explicitly modelling a compliant reaction of the gripping point with respect to a pivoting motion of the grasped object. This is inspired by the fact that, in non-rigid fingertips and/or grippers, it is possible to observe a combination of friction and compliance at the grasping point, when applying an external force to the grasped object. We show that if a robotic manipulator is equipped with finger tips that provide a torsional compliance, a feedback controlled pivoting operation is feasible, provided that a wrench measurement 


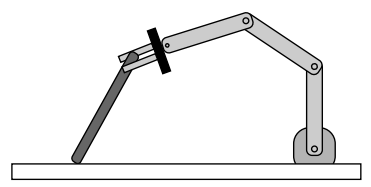

(a)

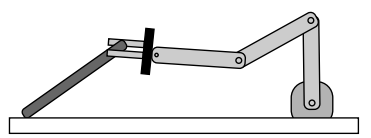

(b)
Fig. 2: Under a rigid grasp, a manipulation task such as reorienting an object against a surface requires significant rearrangement of the manipulator configuration.

is available at the robot wrist. This compliance enables the online estimation of the contact between object and external environment, without compromising the dexterity as in the case of a rigid grasp.

Our method relies on the assumption that a grasp is exerted on an object in such a way that, if force is applied to the object, it will rotate in a compliant manner, generating a torque at the pinching point. This allows us to estimate the pose of the grasped object with respect to an external surface, as well as the contact force. We present a series of experiments designed to showcase the viability of the proposed method, and how adding the possibility of adapting the estimated compliance at the fingertips increases the estimation robustness for a wider range of relative object rotations. We believe that this approach is a promising step towards dexterous manipulation via extrinsic dexterity, in robots equipped with grippers that provide a mixture of compliance and friction at the fingertips.

\section{RELATED WORK}

Classic grasping problems often rely on achieving force closure on an object while optimizing some quality metric [4], thus restraining the object motion. This can be done by using simulators in order to plan a grasp for complex endeffectors [5]. When concerned with dexterous manipulation, however, force closure is not necessarily the desired goal. The robot needs to be able to change the grasp on the object, which can be achieved, for instance via regrasping, which requires discrete primitives of picking and placing, or finger-gaiting, which is an intrinsic dexterous primitive. Other options are, for example, sliding and rolling [1], [2], which exploit the environment, object properties or both in order to facilitate the dexterous task.

The large amount of available options when it comes to grasping and manipulating objects has led to the publication of multiple taxonomies, that provide handy categorizations of grasps with respect to a wide array of purposes. Grasp taxonomies exclude the option of relative motion between object and hand [6], and thus manipulation taxonomies have also been defined, where manipulation tasks are segmented according to features such as contact, deformation, penetration or motion [7], [8].

Extrinsic dexterity has been proposed as a possible mean to navigate between grasp types in a grasp taxonomy by exploiting resources that are extrinsic to the hand, such as the environment or manipulator dynamics [3]. Several articles

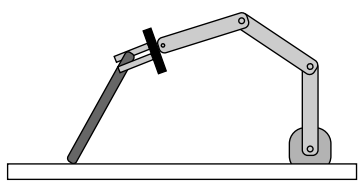

(a)

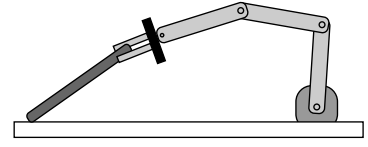

(b)
Fig. 3: If the gripper allows for a rotational degree of freedom between manipulator and grasped object, the same manipulation task can be achieved with smaller joint movements.

make use of this concept in order to approach a dexterous manipulation task with simple grippers, executing pivoting operations through dynamics, or by controlling the object slippage due to gravity by modulation of the grasping force [9]-[11]. If feedback on the object pose is available, it is possible to use dynamic control in order to swing-up a grasped object [12]. More generic dexterous manipulation tasks have been shown in [13], with several types of contact being considered, and with the task being formulated as an optimization problem.

Our work focus on executing a pivoting task by assuming a fingertip that allows for a compliant behavior at the pinching point. This is modelled as an extension of the robotic manipulator via a passive last joint, similar to the systems considered in [14], [15]. In addition, the compliance at the pinching point makes this last joint flexible, similar to [16], [17]. However, in our case the joint state is not observable, as in the gripper proposed in [18]. This type of functional redundancies has been analysed in the context of cooperative manipulation [19], where the passive last joint is considered part of the grasped object ('tool'), and the advantages for this type of manipulation are shown.

Our technique relies on the key assumption that the grasping point allows for the compliant pivoting of the object in the robotic hand. This can be achieved literally through the addition of a torsional spring to, e.g., the gripper in [18]. However, the fundamental requirement for estimating the contact state between object is the presence of a significant contact force between object and surface. Thus, we believe that this technique can be adapted to the case where a soft fingertip is present [20], or the whole gripper might present compliant properties [21], such that significant contact forces are present, and the object is not rigidly attached to the robotic end-effector.

\section{PROBLEM DESCRIPTION}

\section{A. Preliminar assumptions}

We assume planar operation, and that we have a robotic manipulator with $n$ degrees of freedom, such that $n>3$, which is fully or over-actuated in the given plane, equipped with a wrist force/torque sensor and a parallel gripper. A typical parallel gripper will prevent any motion of the grasped object if enough gripping force is applied. If the applied gripping force is insufficient, relative translation 


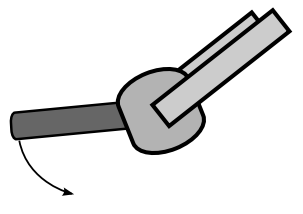

(a)

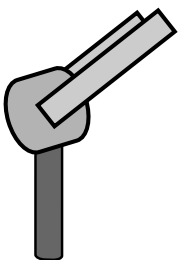

(b)

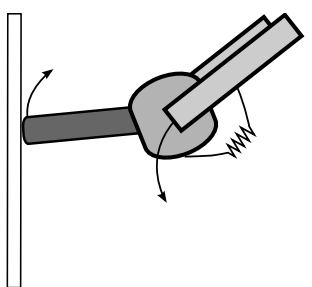

(a)

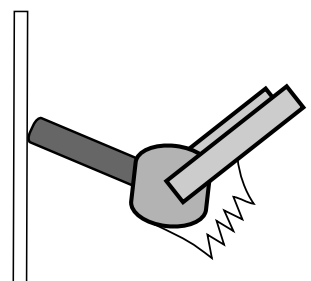

(b)
Fig. 4: A revolute joint at the gripping point allows gravity to reorient an object held by the gripper [18].

and/or rotation might occur between the object and the gripper [12].

Similarly to [18], we assume that the grasped object can freely rotate around the pinching point for a certain range of gripping forces. In addition, we add the constraint that such a revolute degree of freedom is coupled to the parallel gripper through a torsional spring, Fig. 5. The spring provides a torque that is proportional to its displacement from the resting state. This compliant element allows the robot to exert forces on the surface, without the sacrifice in dexterity a rigid grasp would impose.

\section{B. Manipulation task}

Our objective is to control the robotic manipulator so as to regulate the pose of a grasped object with respect to the external surface. Starting from an initial contact state, such can be the case of a robot that reorients the object with respect to its gripper, resulting in a pivoting operation. It can also be that the robot is executing, e.g., an assembly task, and the external surface is an assembly part that requires a certain pose for the grasped object to be assembled.

The revolute joint at the gripping point allows for this goal to be achieved via an independent rotational motion of the grasped object and end-effector, which is a significant distinction from manipulation problems where a rigid grasp is assumed. The added degree-of-freedom allows for more dexterous motions, Fig. 3, and the assumed compliance at the gripping point provides the system with the ability to estimate the state of the contact between the grasped object and the external surface.

\section{Modelling}

The robotic manipulator grasps an object such that there is no linear slippage but only a rotational degree of freedom between the object and end-effector. We consider that the rotational degree of freedom is elastic, that is, there is a torque about the axis connecting the grasping points, which is proportional to the displacement of the rotational joint from a resting position. This is modelled as a torsional spring around the rotational axis. It is assumed that the grasped object is in contact with a rigid surface of known slope. We attach the frame $\{s\}$ at a kinematically known point of the operation plane; the surface frame is defined by the surface normal $\mathbf{n}$ and the tangent vector $\mathbf{s}(\mathbf{n})$, where
Fig. 5: If the grasp is compliant, it is possible to reorient the grasped object by interacting with an external surface.

$\mathbf{s}(\mathbf{n})=\left[-n_{y}, n_{x}\right]^{\top}$ for a vector $\mathbf{n}=\left[n_{x}, n_{y}\right]^{\top}$. Without loss of generality we assume that the surface frame is aligned with the reference frame: $\mathbf{n}=[0,1]^{\top}$.

We will omit the time dependency of all variables, and instead use upper case letters to denote constant scalar values. Let $\mathbf{p}_{c}=\left[x_{c}, y_{c}\right]^{\top}, \mathbf{p}_{e}=\left[x_{e}, y_{e}\right]^{\top}$ be respectively the contact and end-effector position expressed in the surface frame. The orientation of the end-effector and the object with respect to the surface frame are defined by the angles $\theta_{e}$ and $\theta_{c}$ respectively, shown in Fig. 6. The force at the contact point is given by $\mathbf{f}_{c}=\left[f_{c_{x}}, f_{c_{y}}\right]^{\top}$ and at the end-effector by $\mathbf{f}_{e}=\left[f_{e_{x}}, f_{e_{y}}\right]^{\top}$. In the following, we neglect the effect of the grasped-object dynamics on the system, and assume that the end-effector moves sufficiently slow, such that inertial forces are negligible, and that gravity forces are compensated in the measured force and torque.

\section{A. Kinematics}

Let $\mathbf{r}$ be the vector that points from the contact to the gripping point,

$$
\mathbf{r}=\mathbf{p}_{e}-\mathbf{p}_{c} .
$$

By defining $L$ as the distance between the gripping to the contact point we get $\mathbf{r}=\left[L \cos \theta_{c}, L \sin \theta_{c}\right]^{\top}$. The length $L$ is related to the horizontal distance between end-effector and contact point $d_{x}=x_{e}-x_{c}$ as follows:

$$
L=\frac{d_{x}}{\cos \theta_{c}} \text {. }
$$

The differential kinematics of system are given by

$$
\dot{\mathbf{p}}_{e}=\left[\begin{array}{ll}
\mathbf{I}_{2} & \mathbf{s}(\mathbf{r})
\end{array}\right]\left[\begin{array}{c}
\dot{\mathbf{p}}_{c} \\
\dot{\theta}_{c}
\end{array}\right]
$$

where $\mathbf{I}_{2}$ is the identity matrix of dimension 2 . In addition to (2), the contact with the rigid surface imposes the constraint

$$
\dot{y}_{c}=0 \text {. }
$$

From the assumed contact with an external surface, one can derive the expected reaction wrenches by considering the reciprocal of the possible robot movement directions [22]. In case of a rigid grasp, this can be obtained from the surface normal and object pose. However, if rotations are allowed with respect to the gripping point, reaction forces would be expected only for singularities in the mapping (2) 


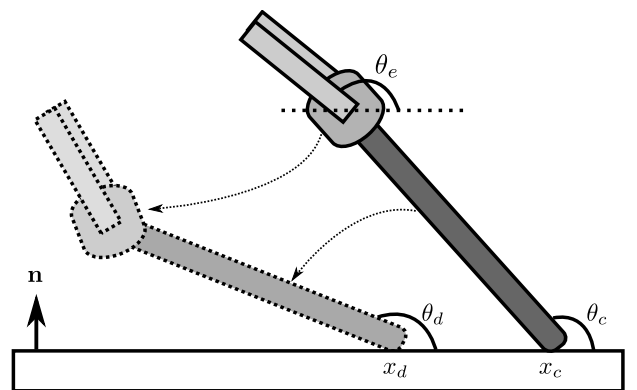

Fig. 6: The manipulation task consists in driving the grasped object to a desired pose $x_{d}, \theta_{d}$.

[23]. The addition of compliance in the gripping points links the exerted forces with the manipulation kinematics, such that the manipulator is able to arbitrarily change its pose without losing the ability to measure the contact state with the surface.

\section{B. Forces}

The grasping point is assumed to provide a rotational degree of freedom to the grasped object, with respect to the gripper. We also assume that this degree of freedom is elastic, that is, there is a torque at the griping point that is proportional to the spring deflection, $\Theta_{o}-\theta_{s}$, where $\Theta_{o}$ defines the spring resting position with respect to the endeffector, and $\theta_{s}$ is the angle of the spring in the surface frame. The torque at the end-effector follows the spring model:

$$
\tau_{e}=K_{s}\left(\Theta_{o}-\theta_{s}\right)
$$

where $K_{s}$ is the spring stiffness coefficient.

The robot is able to deform the spring in two possible ways:

1) Rotation of the end-effector, which changes the spring deformation at a rate $\dot{\theta}_{s^{\prime}}=\dot{\theta}_{e}$.

2) Pivoting of the grasped part, which originates a change $\dot{\theta}_{s^{\prime \prime}}=-\dot{\theta}_{c}$. This is governed by (2) and (3), which establishes the relationship between the translation of the end-effector along the surface normal and the rotation of the grasped part.

By summing both contributions we obtain the deformation rate of the spring,

$$
\dot{\theta}_{s}=\dot{\theta}_{s^{\prime}}+\dot{\theta}_{s^{\prime \prime}}=\dot{\theta}_{e}-\dot{\theta}_{c}
$$

and thus the following expression for the spring angle,

$$
\theta_{s}=\theta_{e}-\theta_{c}+\Delta,
$$

where $\Delta$ is an integration constant, which is defined by the initial grasp on the object such that the torque at the grasping point is zero.

The spring allows the system to exert a force on the rigid surface, $\mathbf{f}_{c}$ with more than just a frictional component. This force is related to the torque at the end-effector (4) as follows:

$$
\tau_{e}=\mathbf{s}(\mathbf{r})^{\top} \mathbf{f}_{c}
$$

where $\mathbf{s}(\mathbf{r})=\left[-L \sin \theta_{c}, L \cos \theta_{c}\right]^{\top}$. It is important to note that the component of $\mathbf{f}_{c}$ along the surface tangent, $f_{c_{x}}$, will, in general, be the resultant of the action of frictional forces on the contact point. The addition of a compliant element at the grasping point allows for the normal element of $\mathbf{f}_{c}, f_{c_{y}}$, to be more prominent.

\section{Manipulation}

We want to move the robotic arm in such a way that its end-effector is able to drive the contact point between the object and the surface to a desired pose, while ensuring contact is maintained, Fig. 6. Let $\mathbf{x}_{c}=\left[\begin{array}{ll}x_{c}, & \theta_{c}, f_{c_{y}}\end{array}\right]^{\top}$ be the task related state, and let the control input for this state be $\mathbf{u}=\dot{\mathbf{x}}_{e}$. Also, consider the augmented state $\overline{\mathbf{x}}_{c}=$ $\left[\mathbf{x}_{c}^{\top}, K_{s}\right]^{\top}$, which includes the torsional stiffness at the grasping point. For this work, we will ignore the frictional component in (6), which is a reasonable assumption for contact between relatively smooth objects. We then have

$$
\begin{aligned}
\dot{\mathbf{x}}_{c} & =\mathbf{J}_{t}\left(\mathbf{x}_{c}\right) \mathbf{u} \\
\mathbf{y} & =\mathbf{h}\left(\overline{\mathbf{x}}_{c}\right)
\end{aligned}
$$

for, respectively, the system process and output equations.

The matrix $\mathbf{J}_{t}\left(\mathbf{x}_{c}\right)$ in the process equation (7) is the task Jacobian, and can be computed from (2), (3) and (4), using the relationship (1) to substitute $L$. We have:

$$
\mathbf{J}_{t}\left(\mathbf{x}_{c}\right)=\left[\begin{array}{ccc}
1 & \tan \theta_{c} & 0 \\
0 & \frac{1}{d_{x}} & 0 \\
0 & \frac{d_{x} \tan \theta_{c} f_{c_{y}}+K_{s}}{d_{x}^{2}} & -\frac{K_{s}}{d_{x}}
\end{array}\right] .
$$

Note that $\mathbf{J}_{t}\left(\mathbf{x}_{c}\right)$ is well-defined for $\left|\mathbf{r}^{\top} \mathbf{n}\right| \neq L$. The output equations are given by

$$
\mathbf{y}=\left[\begin{array}{c}
\frac{\tau_{e}}{f_{e_{y}}} \\
\theta_{e} \\
f_{e_{y}}
\end{array}\right] \quad \mathbf{h}\left(\overline{\mathbf{x}}_{c}\right)=\left[\begin{array}{c}
d_{x} \\
\theta_{c}-\frac{d_{x} f_{c_{y}}}{K_{s}} \\
f_{c_{y}}
\end{array}\right] .
$$

Where we assume that $\Theta_{0}$ and $\Delta$ are accounted for. The measurement equations (10) includes the standard forcetorque relationship (6), but also the spring equation (4). These two equations allow us to relate the object pose with the torque at the end-effector without having to account for friction.

\section{A. Control}

Let $\mathbf{x}_{d}=\left[x_{d}, \theta_{d}, f_{c_{y_{d}}}\right]^{\top}$ be the desired goal state for the system. Under the modelling assumptions, all the force exerted along the surface tangent should result in contact point motion and thus $f_{c_{x}}=0$. We define the control error as $\mathbf{e}=\mathbf{x}_{d}-\mathbf{x}_{c}$. The dynamics of the error, $\dot{\mathbf{e}}$ are obtained through differentiation,

$$
\dot{\mathbf{e}}=-\mathbf{J}_{t}\left(\mathbf{x}_{c}\right) \mathbf{u},
$$

and thus the system (11) can be controlled by choosing

$$
\mathbf{u}=\mathbf{J}_{t}^{-1}\left(\mathbf{x}_{c}\right) \boldsymbol{\Gamma e},
$$




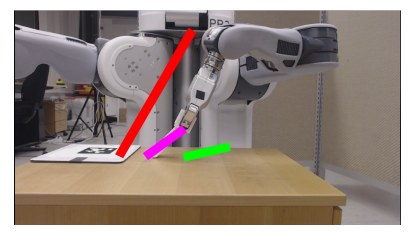

(a)

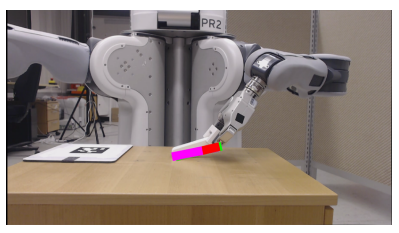

(b)

Fig. 7: Object manipulation with force control. The red bar is the projection of the object pose estimate on the camera frame. The pink bar shows the computed ground truth, and the green bar is the object target pose.

where $\Gamma$ is a positive-definite matrix and

$$
\mathbf{J}_{t}^{-1}\left(\mathbf{x}_{c}\right)=\left[\begin{array}{ccc}
1 & -d_{x} \tan \theta_{c} & 0 \\
0 & d_{x} & 0 \\
0 & \frac{d_{x}}{K_{s}}\left(\frac{K_{s}}{d_{x}}+\tan \theta_{c} f_{c_{y}}\right) & -\frac{d_{x}}{K_{s}}
\end{array}\right],
$$

which is well-defined for $\left|\mathbf{r}^{\top} \mathbf{n}\right| \neq L$, and allows for the feedback linearisation of (7). The closed-loop system becomes

$$
\dot{\mathbf{e}}=-\Gamma \mathbf{e}
$$

which is stable for all positive definite $\Gamma$.

\section{B. Estimation}

The state $\overline{\mathbf{x}}_{c}$ is not directly available, and must be estimated through the measurements $\mathbf{y}$. Let $\hat{\mathbf{x}}_{c}$ be the state estimate. We can estimate the state by employing an Extended Kalman Filter (EKF). Given the observer model

$$
\dot{\hat{\mathbf{x}}}_{c}=\overline{\mathbf{J}}_{t}\left(\hat{\overline{\mathbf{x}}}_{c}\right) \mathbf{u}+\mathbf{K}\left(\hat{\overline{\mathbf{x}}}_{c}\right)\left(\mathbf{y}-\mathbf{h}\left(\hat{\overline{\mathbf{x}}}_{c}\right)\right),
$$

where $\overline{\mathbf{J}}_{t}\left(\overline{\mathbf{x}}_{c}\right)=\left[\begin{array}{c}\mathbf{J}_{t}\left(\mathbf{x}_{c}\right) \\ \mathbf{0}_{3}^{\top}\end{array}\right]$, with $\mathbf{0}_{3}$ being a zero column vector of dimension 3 . We must compute the gain $\mathbf{K}\left(\hat{\overline{\mathbf{x}}}_{c}\right)$ from the process and observation model jacobians, respectively $\mathbf{A}\left(\overline{\mathbf{x}}_{c}\right)$ and $\mathbf{C}\left(\overline{\mathbf{x}}_{c}\right)$ as

$$
\mathbf{K}\left(\hat{\overline{\mathbf{x}}}_{c}\right)=\mathbf{P C}\left(\hat{\overline{\mathbf{x}}}_{c}\right)^{\top} \mathbf{Q}^{-1}
$$

with

$$
\dot{\mathbf{P}}=\mathbf{A}\left(\hat{\overline{\mathbf{x}}}_{c}\right) \mathbf{P}+\mathbf{P A}\left(\hat{\overline{\mathbf{x}}}_{c}\right)^{\top}+\mathbf{R}-\mathbf{P C}\left(\hat{\overline{\mathbf{x}}}_{c}\right)^{\top} \mathbf{Q}^{-1} \mathbf{C}\left(\hat{\overline{\mathbf{x}}}_{c}\right) \mathbf{P},
$$

where the matrices $\mathbf{R}$ and $\mathbf{Q}$ model the uncertainty in the system (7) and (8), which is assumed to be represented by additive Gaussian noise. The process model Jacobian is given by

$$
\begin{aligned}
& \mathbf{A}\left(\overline{\mathbf{x}}_{c}\right)=\frac{\partial \overline{\mathbf{J}}_{t}\left(\overline{\mathbf{x}}_{c}\right) \mathbf{u}}{\partial \overline{\mathbf{x}}_{c}}= \\
& {\left[\begin{array}{cccc}
0 & \frac{\dot{y}_{e}}{\cos ^{2} \theta_{c}} & 0 & 0 \\
\frac{\dot{y}_{e}}{d_{x}^{2}} & 0 & 0 & 0 \\
\frac{d_{x} \gamma-K_{s} \dot{y}_{e}}{d_{x}^{3}} & \frac{f_{c_{y}} \dot{y}_{e}}{d_{x} \cos ^{2} \theta_{c}} & \frac{\tan \theta_{c} \dot{y}_{e}}{d_{x}} & \frac{\dot{y}_{e}-d_{x} \dot{\theta}_{e}}{d_{x}^{2}}
\end{array}\right],}
\end{aligned}
$$

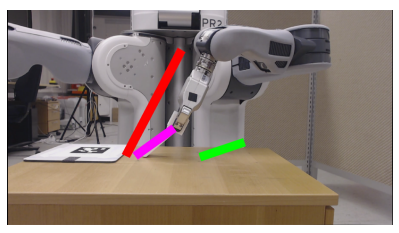

(a)

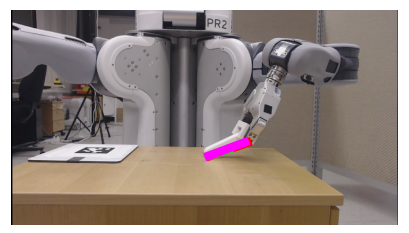

(b)
Fig. 8: Object manipulation without force control. The robot pivots the object into the desired pose, without rotating its end-effector.

\begin{tabular}{|c|c|c|c|}
\hline Parameter & Value & Parameter & Value \\
\hline $\mathbf{Q}$ & $\operatorname{diag}(200,10,5 \mathrm{e} 3)$ & $x_{d}$ & $0.2 \pm 0.05 \mathrm{~m}$ \\
\hline $\mathbf{R}_{\text {no adapt }}$ & $\operatorname{diag}\left(10^{-2}, 10^{-2}, 10^{-3}, 0\right)$ & $\theta_{d}$ & $0.3 \pm 0.05 \mathrm{rad}$ \\
\hline $\mathbf{R}_{\text {adapt }}$ & $\operatorname{diag}\left(10^{-2}, 10^{-2}, 10^{-3}, 3 \mathrm{e}-5\right)$ & $\mathbf{P}_{\text {init }}$ & $0.1 \mathbf{I}$ \\
\hline $\boldsymbol{\Gamma}_{\text {manip }}$ & $\operatorname{diag}(1.0,1.0,0.7)$ & $f_{d}$ & $-1.25 \pm 0.2 \mathrm{~N}$ \\
\hline $\boldsymbol{\Gamma}_{\text {pivot }}$ & $\operatorname{diag}(0.7,0.7,0)$ & $K_{s}$ & $0.17 \mathrm{~N} . \mathrm{m} / \mathrm{rad}$ \\
\hline
\end{tabular}

TABLE I: Estimator and controller parameters for the manipulation and pivoting experiments, with the range for the desired values. $\mathbf{R}_{\text {adapt }}$ refers to the assumed process model covariance with stiffness adaptation, and $\mathbf{R}_{\text {no adapt }}$ refers to the one without it. $\boldsymbol{\Gamma}_{\text {manip }}$ and $\boldsymbol{\Gamma}_{\text {pivot }}$ refer respectively to the controller gains for the manipulation and pivoting experiments.

where $\gamma=\tan \theta_{c} f_{c_{y}} \dot{y}_{e}-K_{s} \dot{\theta}_{e}$. The observation model Jacobian is then given by

$$
\mathbf{C}\left(\overline{\mathbf{x}}_{c}\right)=\frac{\partial \mathbf{h}\left(\overline{\mathbf{x}}_{c}\right)}{\partial \overline{\mathbf{x}}_{c}}=\left[\begin{array}{cccc}
-1 & 0 & 0 & 0 \\
\frac{f_{c_{y}}}{K_{s}} & 1 & -\frac{d_{x}}{K_{s}} & \frac{d_{x} f_{c_{y}}}{K_{s}^{2}} \\
0 & 0 & 1 & 0
\end{array}\right] .
$$

By performing the estimation on the augmented system state $\overline{\mathbf{x}}_{c}$, we allow the system to adapt the constant $K_{s}$, adding robustness against minor modelling errors and inaccurate initial guess for the stiffness at the grasping point.

\section{EXPERIMENTAL EVALUATION}

\section{A. Experimental setup}

Our experimental setup consists of a PR2 robot equipped with wrist force/torque sensors, positioned close to a table, Fig. 1. The surface frame is obtained through a fiducial system [24] that detects the marker laid on the surface. The initial pose of the end-effector is also defined with respect to this marker.

In order to obtain a compliant grasp, we 3D-printed an object that consists on a revolute joint, on which two torsional springs provide the compliance. The robot is able to rigidly grasp this object around the rotational axis, thus creating the conditions modelled in (4) and (5), with $\Theta_{0}=\Delta=0$.

We define two experiments: a) regulation of the object pose and b) object pivoting. In the following sections, we illustrate the control and estimation performance on 20 iterations of the experiments, and show how allowing for adaptation in the value of $K_{s}$ affects the overall system performance. Ground truth data is obtained by intersecting a circle of radius $L$, centered at the grasping point, with a line along the surface tangent. In all the experiments the endeffector starts at a pre-defined pose in the surface frame and approaches the surface along its normal until a force of $1 \mathrm{~N}$ 

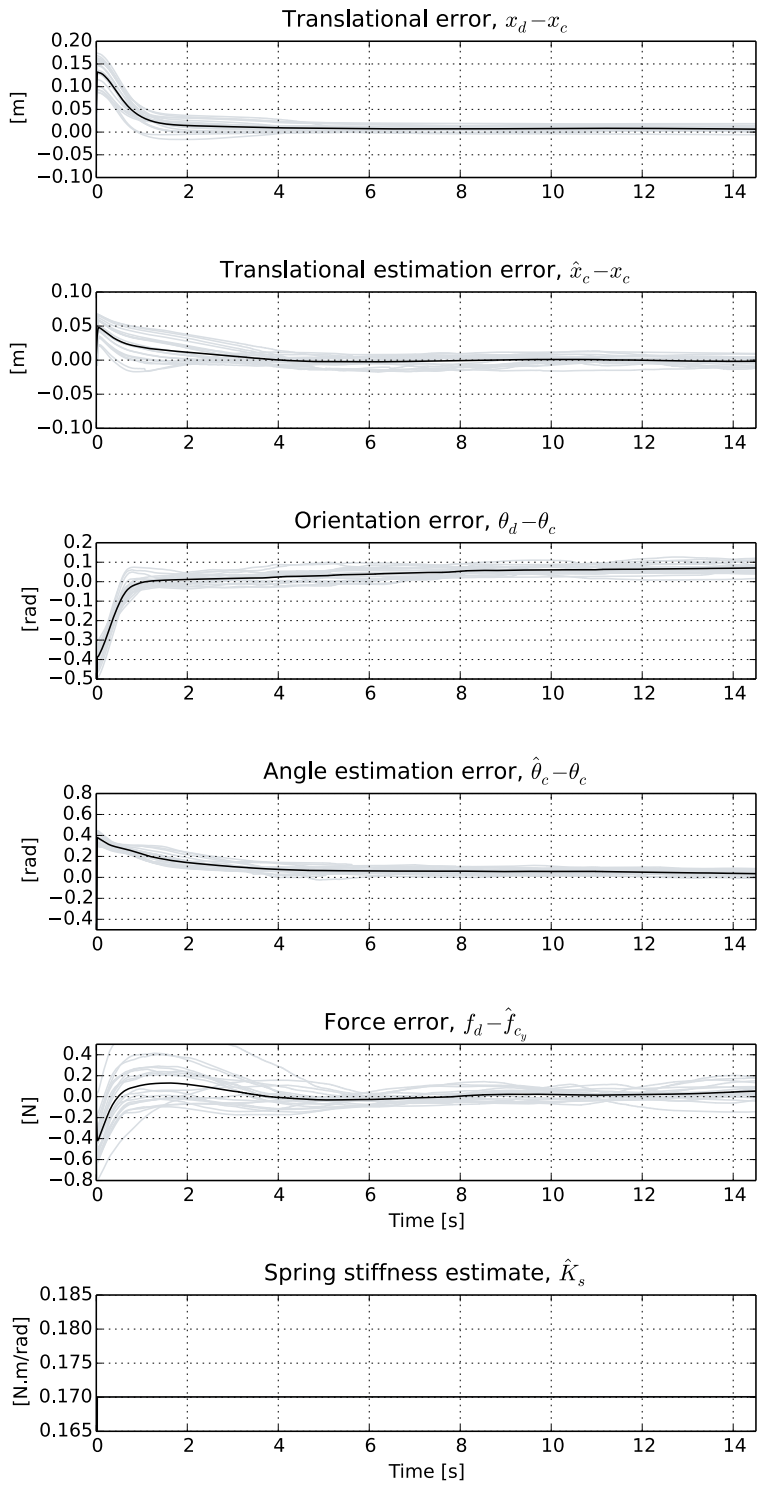

Fig. 9: Results obtained for the manipulation experiments, without stiffness adaptation. Each grey plot is the result of one experiment execution, while the black plot represents the average of all the consecutive runs.

is detected. Each experiment was run 20 consecutive times for 15 seconds.

\section{B. Regulating the object pose}

In this experiment, we evaluate the system in its ability to attain the control objective stated in section V-A. We executed the manipulation task with and without stiffness adaptation, respectively Fig. 9 and Fig. 10. In both cases the desired state is drawn from a uniform distribution as in Table I. The force estimation error is not shown in the plots, as the raw data noise dominates its value. Instead, we illustrate the estimator performance by plotting the force estimate with respect to the raw values for one of the runs, Fig. 13.

Note that, from (4) and (6), it is clear that we must have
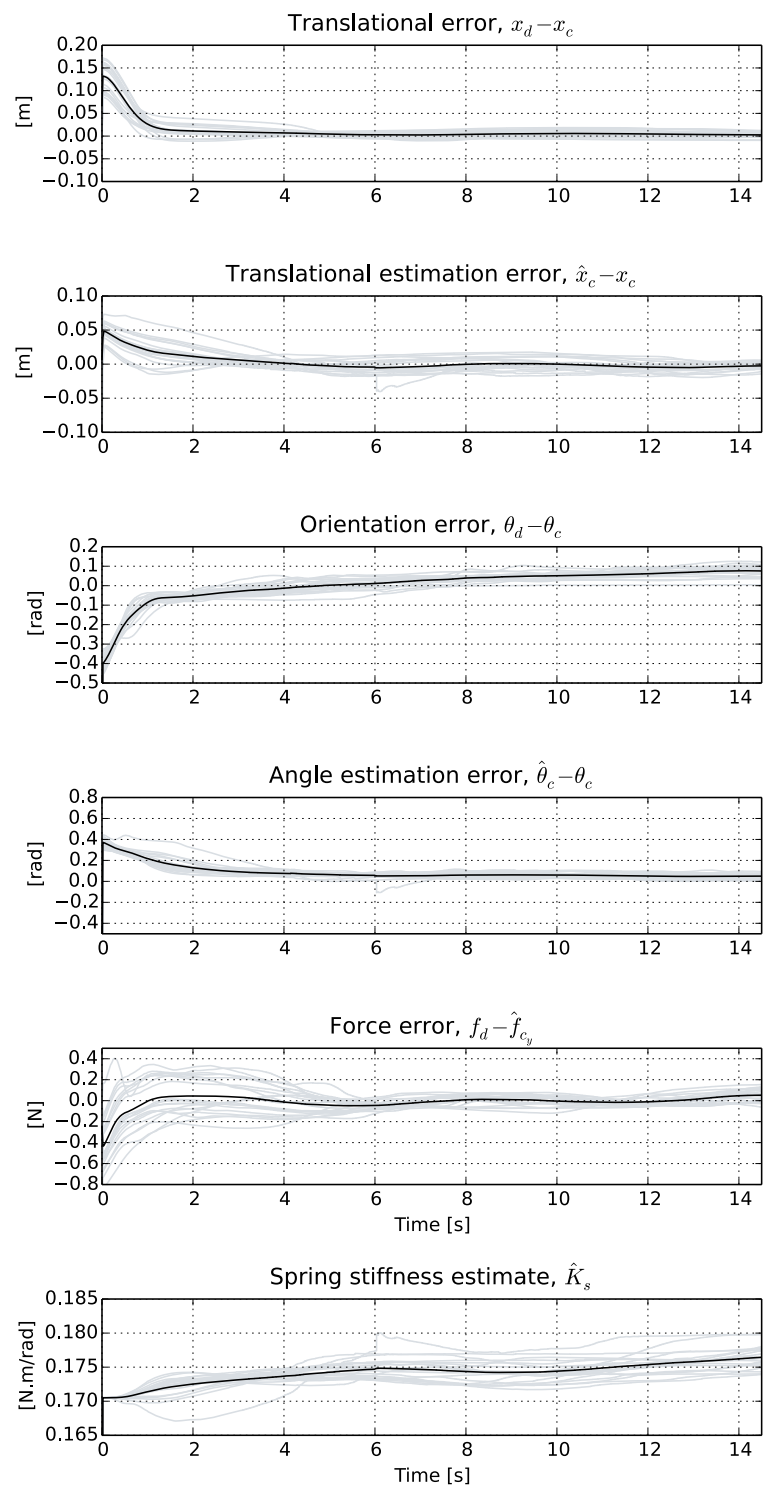

Fig. 10: When the system is controlling the contact force, the torque at the gripping point can be maintained within pre-determined ranges. When the stiffness can be previously estimated for those ranges, there is no clear advantage in allowing the system to adapt the spring constant.

$\theta_{s}=\Theta_{0}-\mathbf{s}(\mathbf{r})^{\top} \mathbf{f}_{d} / K_{s}$ in order to get $f_{c_{y}}=f_{d}$, with $\mathbf{f}_{d}=\left[0, f_{d}\right]^{\top}$. Thus, if we control the contact force, $\theta_{s}$ will stay in a range related to the range of desired contact forces. In a scenario where it is possible to estimate the stiffness at the grasping point for predictable values of $\theta_{s}$, there is no need for adaptation. In fact, for the initial value of $K_{s}=$ $0.17 \mathrm{~N} . \mathrm{m} / \mathrm{rad}$ used as the initial stiffness value in all the experiments, there is no significant change in the results with and without adaptation. In both cases we observed a final error of about $2 \mathrm{~mm}$ for $x_{c}$ and 0.04 radians for $\theta_{c}$.

\section{Pivoting}

This experiment evaluates the case where the manipulation goal is the grasped object reorientation with respect to the 

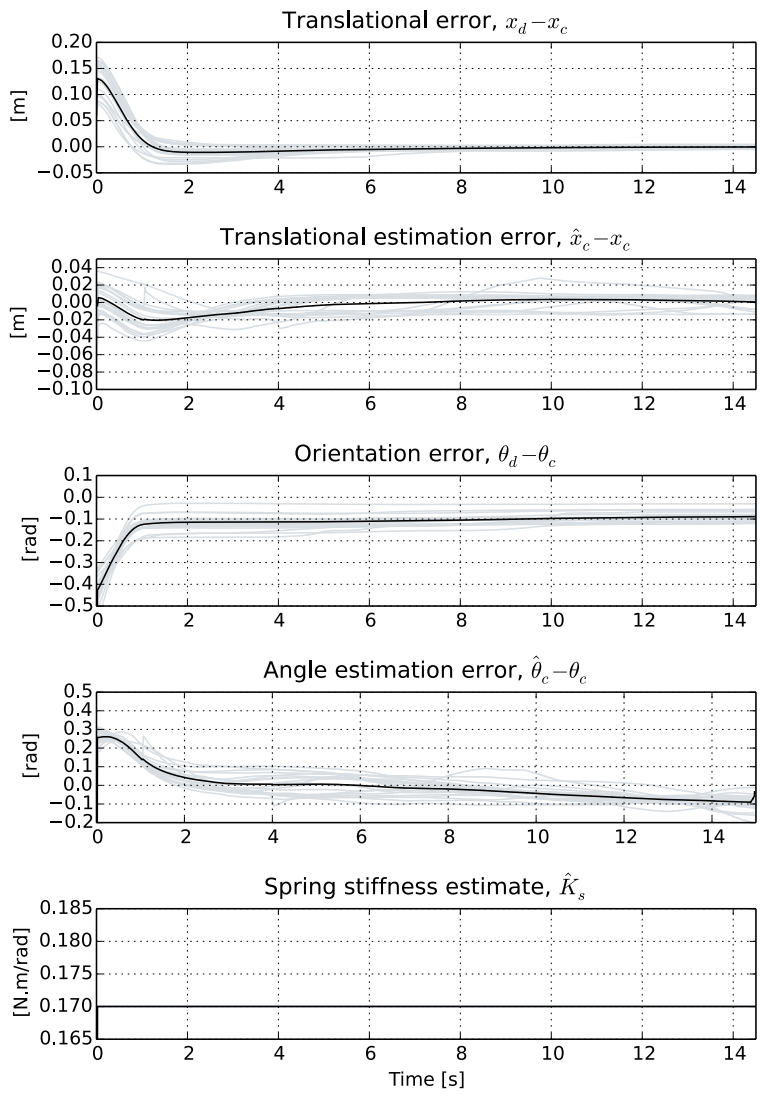

Fig. 11: The proposed method can be used for pivoting the grasped object. In this scenario, the experiments were run without the force compensation component of the controller. This leads to larger spring deflections, which reduces the accuracy of the spring model (4).

end-effector. The system (7) is fully actuated, and thus the contact force cannot be regulated independently from the end-effector orientation. Consequently, in order to pivot the object, we impose $\dot{\theta}_{e}=0$. A practical implementation could limit the force as $\hat{f}_{c}, f_{\text {lower }} \leq \hat{f}_{c} \leq f_{\text {upper }}$ such that if the force estimate moves out of these bounds, the full controller is activated, ensuring that contact is kept. Note that there is a clear trade-off in achieving force control, Fig. 7, or taking advantage of the rotational degree-of-freedom at the grasping point in order to perform a more dexterous motion, Fig. 8 .

We execute the pivoting operation firstly without spring adaptation, and then allowing spring adaptation, respectively Fig. 11 and 12. In these experiments, it becomes clear that for the same value of $K_{s}$ as in section VI-B the angle estimation is biased. In fact, while pivoting, the spring deflection will be larger, and the measured torque will most likely not follow the simple model (4), due to the absence of force control. By enabling the stiffness adaptation, Fig. 12, we obtain better results. The average orientation error for the experiment without stiffness adaptation is of 0.09 radians, while with spring adaptation the average error fell to 0.02 radians.

Additionally, we can observe that the overall performance
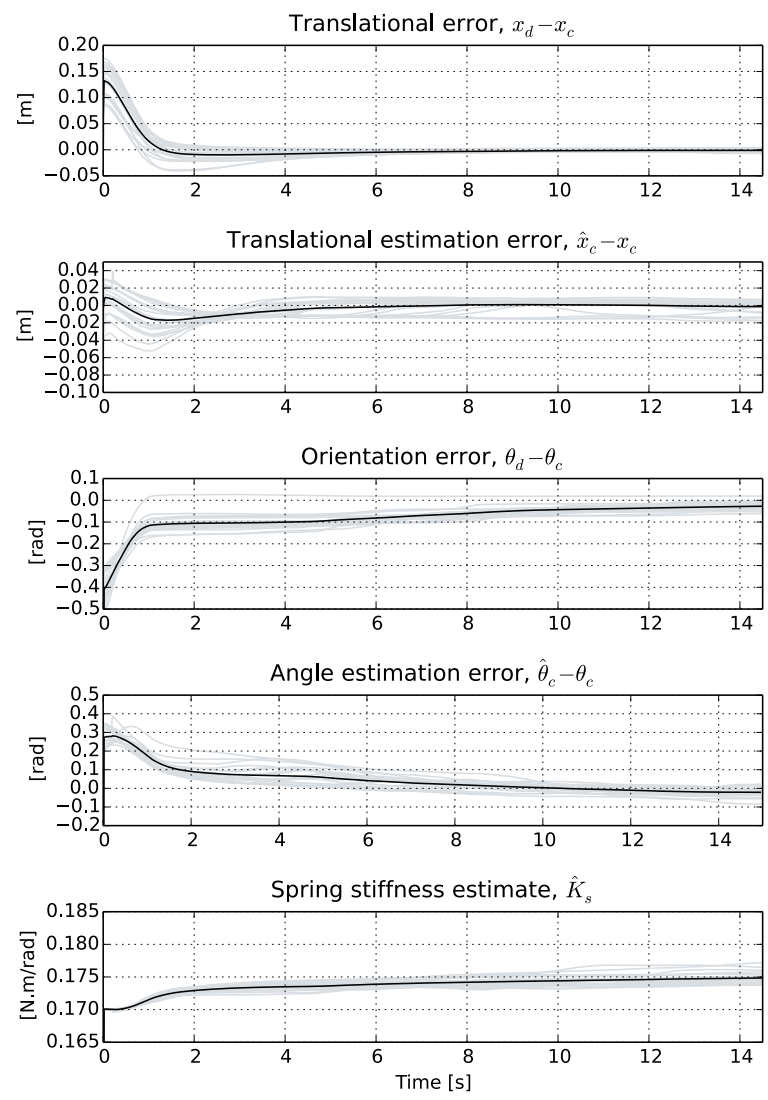

Fig. 12: By allowing the estimator to change the value of the modelled stiffness constant, better results are achieved.

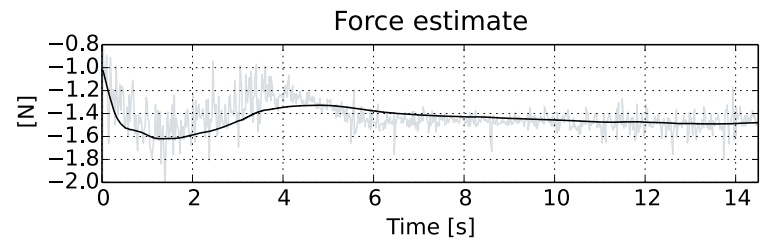

Fig. 13: Force estimation results. The black line is the force estimate $\hat{f}_{c_{y}}$, while the gray plot depicts the measured $f_{e_{y}}$.

of the system for the pivoting experiments is smoother. This can be explained by that fact that a vertical displacement of the end-effector will change the contact force, according to (9) and, due to the compliant nature of the PR2 arm, precise fine motion of the end-effector is not always possible, leading to some conflict between the contact force regulation and the pose alignment of the grasped object.

\section{CONCLUSION}

In this work we presented a technique for dexterous manipulation of an object under a compliant grasp. We showed that, for scenarios where it is reasonable to assume that the grasping point of the end-effector offers some compliance around a rotational axis, the orientation of the grasped object is controllable independently of the end-effector orientation. 
This is a significant distinction from typical manipulation tasks where a perfectly rigid grasp is assumed [26]-[28].

The method employs an EKF in order to produce estimates of the contact point state. It relies only on knowledge of the contact surface and on the availability of force/torque measurements at the robot's wrist. Furthermore, we allow for the estimator to adapt the value of the modelled gripping stiffness, adding some robustness to deviations from the employed linear model. We believe that the presented method could be straightforwardly implemented in a robot equipped with a gripper such as the presented in [18], where the rotational finger tips are made compliant.

A robot equipped with soft fingertips can potentially exert sufficient forces with a grasped object, while the object is able to move relative to the end-effector. Modulating the gripping pressure can also change the friction forces and the compliant reaction between object and end-effector, which would introduce an additional degree of freedom for the control problem. The present work can be adapted to prevent the stiffness adaptation to converge to non-physical values, as in [29]. Furthermore, including friction in the estimation model could potentially reduce the dependency on the used spring model to estimate the object angle with respect to the surface.

\section{REFERENCES}

[1] A. M. Okamura, N. Smaby, and M. R. Cutkosky. An overview of dexterous manipulation. In Proceedings 2000 ICRA. Millennium Conference. IEEE International Conference on Robotics and Automation. Symposia Proceedings (Cat. No.00CH37065), volume 1, pages 255262 vol.1, 2000.

[2] A. Bicchi. Hands for dexterous manipulation and robust grasping: a difficult road toward simplicity. IEEE Transactions on Robotics and Automation, 16(6):652-662, Dec 2000.

[3] N. C. Dafle, A. Rodriguez, R. Paolini, B. Tang, S. S. Srinivasa, M. Erdmann, M. T. Mason, I. Lundberg, H. Staab, and T. Fuhlbrigge. Extrinsic dexterity: In-hand manipulation with external forces. In 2014 IEEE International Conference on Robotics and Automation (ICRA), pages 1578-1585, May 2014.

[4] C. Ferrari and J. Canny. Planning optimal grasps. In Proceedings 1992 IEEE International Conference on Robotics and Automation, pages 2290-2295 vol.3, May 1992.

[5] A. T. Miller, S. Knoop, H. I. Christensen, and P. K. Allen. Automatic grasp planning using shape primitives. In 2003 IEEE International Conference on Robotics and Automation (Cat. No.03CH37422), volume 2, pages 1824-1829 vol.2, Sept 2003.

[6] Thomas Feix, Roland Pawlik, Heinz-Bodo Schmiedmayer, Javier Romero, and Danica Kragic. A comprehensive grasp taxonomy. In Robotics, Science and Systems Conference: Workshop on Understanding the Human Hand for Advancing Robotic Manipulation, pages 2-3, 2009.

[7] D. Leidner, C. Borst, A. Dietrich, M. Beetz, and A. Albu-Schäffer. Classifying compliant manipulation tasks for automated planning in robotics. In 2015 IEEE/RSJ International Conference on Intelligent Robots and Systems (IROS), pages 1769-1776, Sept 2015.

[8] I. M. Bullock, R. R. Ma, and A. M. Dollar. A hand-centric classification of human and robot dexterous manipulation. IEEE Transactions on Haptics, 6(2):129-144, April 2013.

[9] A. Holladay, R. Paolini, and M. T. Mason. A general framework for open-loop pivoting. In 2015 IEEE International Conference on Robotics and Automation (ICRA), pages 3675-3681, May 2015.

[10] F. E. Viña B., Y. Karayiannidis, K. Pauwels, C. Smith, and D. Kragic. In-hand manipulation using gravity and controlled slip. In 2015 IEEE/RSJ International Conference on Intelligent Robots and Systems (IROS), pages 5636-5641, Sept 2015.
[11] F. E. Viña B., Y. Karayiannidis, C. Smith, and D. Kragic. Adaptive control for pivoting with visual and tactile feedback. In 2016 IEEE International Conference on Robotics and Automation (ICRA), pages 399-406, May 2016.

[12] A. Sintov, O. Tslil, and A. Shapiro. Robotic swing-up regrasping manipulation based on the impulse x2013; momentum approach and clqr control. IEEE Transactions on Robotics, 32(5):1079-1090, Oct 2016.

[13] Alberto Rodriguez and Nikhil Chavan-dafle. Prehensile Pushing : Inhand Manipulation with Push-Primitives. In International Conference on Intelligent Robots and Systems, 2015.

[14] H. Arai and S. Tachi. Position control of manipulator with passive joints using dynamic coupling. IEEE Transactions on Robotics and Automation, 7(4):528-534, Aug 1991.

[15] Alessandro De Luca and Giuseppe Oriolo. Trajectory planning and control for planar robots with passive last joint. The International Journal of Robotics Research, 21(5-6):575-590, 2002.

[16] S. K. Agrawal and V. Sangwan. Differentially flat designs of underactuated open-chain planar robots. IEEE Transactions on Robotics, 24(6):1445-1451, Dec 2008.

[17] V. Sangwan, H. Kuebler, and S. K. Agrawal. Differentially flat design of under-actuated planar robots: Experimental results. In 2008 IEEE International Conference on Robotics and Automation, pages 24232428, May 2008.

[18] N. Chavan-Dafle, M. T. Mason, H. Staab, G. Rossano, and A. Rodriguez. A two-phase gripper to reorient and grasp. In 2015 IEEE International Conference on Automation Science and Engineering (CASE), pages 1249-1255, Aug 2015.

[19] Pål Johan From and Jan Tommy Gravdahl. Considering passive joints in cooperative manipulation as functional redundancy. $\{$ IFAC Proceedings Volumes, 41(2):4349 - 4354, 2008. 17th \{IFAC\} World Congress.

[20] K.B. Shimoga and A.A. Goldenberg. Soft robotic fingertips. The International Journal of Robotics Research, 15(4):320-334, 1996.

[21] Eric Brown, Nicholas Rodenberg, John Amend, Annan Mozeika, Erik Steltz, Mitchell R. Zakin, Hod Lipson, and Heinrich M. Jaeger. Universal robotic gripper based on the jamming of granular material. Proceedings of the National Academy of Sciences, 107(44):1880918814, 2010.

[22] A. De Luca and C. Manes. Modeling of robots in contact with a dynamic environment. IEEE Transactions on Robotics and Automation, 10(4):542-548, Aug 1994.

[23] Diogo Almeida and Yiannis Karayiannidis. Folding assembly by means of dual-arm robotic manipulation. In 2016 IEEE International Conference on Robotics and Automation (ICRA), pages 3987-3993, May 2016.

[24] Edwin Olson. AprilTag: A robust and flexible visual fiducial system. In Proceedings of the IEEE International Conference on Robotics and Automation (ICRA), pages 3400-3407. IEEE, May 2011.

[25] J. D. Hunter. Matplotlib: A 2d graphics environment. Computing In Science \& Engineering, 9(3):90-95, 2007.

[26] Y. Karayiannidis, C. Smith, F. E. Viña, P. Ögren, and D. Kragic. Model-free robot manipulation of doors and drawers by means of fixed-grasps. In 2013 IEEE International Conference on Robotics and Automation, pages 4485-4492, May 2013.

[27] Georg Bätz, Bernhard Weber, Michael Scheint, Dirk Wollherr, and Martin Buss. Dynamic contact force/torque observer: Sensor fusion for improved interaction control. The International Journal of Robotics Research, 32(4):446-457, 2013.

[28] Y. Karayiannidis, C. Smith, F. E. Viña, and D. Kragic. Online contact point estimation for uncalibrated tool use. In 2014 IEEE International Conference on Robotics and Automation (ICRA), pages 2488-2494, May 2014.

[29] D. Verscheure, J. Swevers, H. Bruyninckx, and J. De Schutter. Online identification of contact dynamics in the presence of geometric uncertainties. In 2008 IEEE International Conference on Robotics and Automation, pages 851-856, May 2008. 